\title{
GERIATRIC SYNDROMES AND NOT CARDIOVASCULAR RISK FACTORS ARE ASSOCIATED WITH COGNITIVE IMPAIRMENT AMONG MEXICAN COMMUNITY-DWELLING ELDERLY WITH TYPE 2 DIABETES
}

\author{
Omar Yaxmehen Bello-Chavolla ${ }^{1}$, Carlos Alberto Aguilar-Salinas ${ }^{2}$ and José Alberto Avila-Funes ${ }^{3,4}$ \\ ${ }^{1} \mathrm{MD} /$ PhD Programme, Facultad de Medicina, Universidad Nacional Autónoma de México; ${ }^{2}$ Department of \\ Endocrinology and Metabolism, and ${ }^{3}$ Geriatrics Department, Instituto Nacional de Ciencias Médicas y Nutrición \\ Salvador Zubirán, Mexico City, Mexico. ${ }^{4}$ Univ. Bordeaux, Inserm, Bordeaux Population Health Research Center, UMR \\ 1219, F-33000, Bordeaux, France
}

\begin{abstract}
Background: The association of cognitive impairment and type 2 diabetes has been consistently shown in several studies, yet its association with geriatric syndromes has not been fully explored. Objective: To study the correlates of cognitive impairment among community-dwelling elderly with type 2 diabetes. Methods: Cross-sectional study of 135 diabetic persons aged 70 years or older participating in the Coyoacán Cohort Study in Mexico City. Baseline data included chronic illnesses, geriatric syndromes, and diabetes-related variables. The lowest quartile in both the Mini-Mental State Examination and the Isaacs Set Test, according to age and schooling, was used to identify participants with cognitive impairment. Multivariate logistic regression analyses were used to identify the correlates of cognitive impairment. Results: Mean age of participants was $77.7 \pm 5.8$ years. The prevalence of cognitive impairment was $14.1 \%$. Univariate logistic regression analyses showed that diabetic nephropathy, depression symptoms, falls, and frailty were associated with cognitive impairment. Multivariate logistic regression analyses showed that urinary incontinence and frailty were independently associated with cognitive impairment. Cardiovascular risk factors and diabetes-related variables did not show significant association to cognitive impairment. Conclusions: Geriatric syndromes, but not cardiovascular risk factors, were independently associated with cognitive impairment among diabetic elderlies. Intentional evaluation of these conditions may be important to improve management of the elderly patient with type 2 diabetes and cognitive impairment. (REV INVES CLIN. 2017;69:166-72)
\end{abstract}

Key words: Geriatric syndrome. Cognitive impairment. Frailty. Urinary incontinence. Diabetes.

Corresponding author:

José Alberto Ávila-Funes

Department of Geriatrics

Instituto Nacional de Ciencias Médicas

y Nutrición Salvador Zubirán

Vasco de Quiroga, 15

Col. Belisario Domínguez Sección XVI, Del. Tlalpan

C.P. 14080, Ciudad de México, México

E-mail: avilafunes@live.com.mx

Received for publication: 09-02-2017

Accepted for publication: 16-03-2017 


\section{INTRODUCTION}

Type 2 diabetes mellitus (DM) is a significant public health burden worldwide, particularly in countries under epidemiological transition ${ }^{1-3}$. Care and management in elderly DM patients is complicated, especially given the simultaneous presence of comorbidity and geriatric syndromes. Of special importance is cognitive impairment (CIM), which is associated with reduced self-care and monitoring of DM as well as higher hospitalization records, among others ${ }^{3}$. The association between DM and CIM has been persistently reported in several studies. In comparison with non-diabetic populations, those with DM have more frequent cognitive impairment ${ }^{4,5}$. In addition, factors such as long evolution of DM, elevated $\mathrm{HbA1c}$ levels, or history of insulin treatment are considered risk factors for the development of CIM. Previous work has demonstrated that Mexican-American participants with DM had a greater risk of dementia and CIM in comparison with non-diabetic participants ${ }^{6-8}$. Similarly, DM patients also have a higher risk of geriatric conditions, including dementia, disability, falls, and urinary incontinence ${ }^{9}$. Despite the large evidence supporting these associations, the relation of geriatric conditions with the presence of CIM in DM patients is still largely unknown; furthermore, previous work suggests that cardiovascular risk factors have a more established role in association with $\mathrm{CIM}^{10}$. Therefore, this study aimed to determine the geriatric and cardiovascular correlates of CIM among community dwelling elderly diabetics.

\section{METHODS}

\section{Study population}

This is a cross-sectional analysis of a subset of participants from the Coyoacán Cohort, an observational study conducted in Mexico City. Specific details for the design of this study have been published elsewhere ${ }^{10}$. Briefly, to be eligible for recruitment, participants had to meet the following criteria: age 70 years or older, established residence in Coyoacán, not being institutionalized, and being registered at the Food Support, Medical Care and Free Drugs Program, which is a government program that includes $95 \%$ of the community dwelling elderly ( $\geq 70$ years of age) in Mexico City. The first phase was composed of a face-to-face interview during which a wide range of information was collected, including self-reported data regarding sociodemographic characteristics, general health-related information, medication use, oral health (self-reported and clinically evaluated), and mental health. Each participant underwent a comprehensive geriatric assessment that included physical performance tests, cognitive tests, and nutrition and medical assessment as well. The Human Research Local Ethics Committee approved all proceedings regarding this study.

\section{Sample}

Patients with type 2 DM were selected from the Coyoacán Cohort and included in this study. Diabetes was defined as the self-report of previously diagnosed DM or self-report of taking DM medications. All considered participants underwent a Mini-Mental State Examination (MMSE) and a semantic verbal fluency test (Isaacs Set Test, IST). Cognitive impairment was defined as a score below the $25^{\text {th }}$ percentile for this specific population, according to age and schooling in both tests, without functional impairment ${ }^{10}$. Patients who only completed either the MMSE or the IST were excluded from the study; no significant differences were found between patients included and excluded from the analysis.

\section{Definition of potential correlates}

Participants were categorized as "frail" if they fulfilled three or more frailty criteria; otherwise if they fulfilled one or two, they were considered to be pre-frail or non-frail if none ${ }^{11}$ :

- Unintentional weight loss of $5 \mathrm{~kg}$ or more in the last 12 months;

- Exhaustion was assessed by the positive answer to two questions from the Center for Epidemiologic Studies-Depression scale (CES-D) :"I felt that everything I did was an effort" and "I could not get going";

- Low physical activity was defined according to the lowest quintile (adjusted by sex) on the Physical Activity Scale for the Elderly questionnaire (PASE);

- Slowness was defined if participants answered "yes" or "can't do" to any of the following two questions: Because of a health problem, "do you have difficulty 
walking one block?" or alternatively, "Do you have difficulty with climbing several flights of stairs without resting?"; and

- Weakness was determined among participants who answered "yes" to the question, "Because of a health problem, do you have difficulty with lifting or carrying objects weighting over $5 \mathrm{~kg}$, like a heavy bag of groceries?".

These definitions have previously been validated for this population ${ }^{12}$.

Fall syndrome was defined as having $>2$ fall episodes in the previous 12 months ${ }^{13}$.

Depressive symptoms were determined when participants had a Geriatric Depression Scale score $>5$ (15-item version) ${ }^{14}$.

Polypharmacy was defined as taking $>3$ different medications at the time of the study ${ }^{15}$.

Urinary incontinence, visual deficit, myocardial infarction, stroke, hypertension, hypercholesterolemia, hypertriglyceridemia, osteoporosis, and smoking were determined by the self-report of each entity and considered individually as binary outcomes.

For diabetes-related variables, duration of DM from diagnosis in years as well as age at diagnosis were each individually assessed; self-report of diabetic nephropathy and diabetic retinopathy was assessed independently and then combined under "Any microvascular complications" for purposes of the analysis. Previous history of insulin use was treated as a binary variable according to self-report by the patient. Macrovascular complications include self-report of diabetic foot or peripheral vascular disease.

Body mass index (BMl; weight/height ${ }^{2}$ ) was also calculated and included as a correlate.

Sociodemographic variables included age (years), sex, and schooling (education grade).

\section{Statistical analysis}

Characteristics of participants were described using arithmetic means and standard deviations (SD) or frequencies and proportions where appropriate. The following statistical procedures were used according to analyzed variables: chi-squared test for qualitative variables, Student $t$-test, and the $U$ of Mann-Whitney tests were used where applicable for quantitative variables. In order to develop an explanatory model for CIM, we fitted multivariate logistic regression models, including several variable-blocks: sociodemographic variables, diabetes-related variables, cardiovascular risk factors, and geriatric conditions. Wald tests were used to eliminate from every model those variables judged not significant at the $20 \%$ level, and then the variables considered significantly associated with CIM were retained. Secondly, a new model including the variables significantly associated with CIM from previous models was run and the cut-off level at this time was $5 \%$ in order to select a set of variables to be included in a last full model. All comparisons were evaluated using $95 \%$ confidence intervals $(\mathrm{Cl})$ and the Nagelkerke $\mathrm{R}^{2}$ was also reported. Statistical analyses were performed in SPSS software for Windows $^{\oplus}$ (SPSS Inc., Chicago, IL, version 19.0).

\section{RESULTS}

The study sample included 135 participants. Mean age was 77.7 years (SD: 5.8 ) and $54.1 \%$ were female. Mean age of DM onset was 62.4 years (SD: 12.5 ), with a mean DM duration of 14.7 years (SD: 11.3), $19.3 \%$ were using insulin treatment, and $57 \%$ reported having at least one microvascular complication (28.1\% diabetic nephropathy and $44.1 \%$ diabetic retinopathy). Of the participants, $18 \%$ had urinary incontinence, $46.7 \%$ had at least two falls in the last year, $20 \%$ reported depressive symptoms, $14 \%$ were frail, and $57.8 \%$ reported currently taking $>3$ medications. Only 19 participants (14.1\%) had CIM.

Table 1 presents the comparative analysis between participants according to the presence or absence of CIM. In comparison to subjects without CIM, those cognitively impaired had more diabetic nephropathy $(p=0.014)$ or the presence of any microvascular complication ( $p=0.046)$, falls $(p=0.047)$, depressive symptoms ( $p=0.013)$, and frailty $(p=0.011)$. The frequency of cardiovascular risk factors, including myocardial infarction, stroke, systemic hypertension, and hypercholesterolemia were not different between groups. 
Table 1. Comparative analysis among participants without or with cognitive impairment

\begin{tabular}{|c|c|c|c|c|}
\hline Variable & $\begin{array}{c}\text { Overall } \\
(n=135)\end{array}$ & $\begin{array}{l}\text { Without cognitive } \\
\text { impairment } \\
(n=116)(\%)\end{array}$ & $\begin{array}{l}\text { With cognitive } \\
\text { impairment } \\
(n=19)(\%)\end{array}$ & $p$ value \\
\hline Women (\%) & $73(54.1)$ & $63(54.3)$ & $10(52.9)$ & 0.892 \\
\hline Age (Mean \pm SD) & $77.7 \pm 5.8$ & $77.7 \pm 5.7$ & $77.5 \pm 7.1$ & 0.414 \\
\hline Years of schooling (Median \pm IQR) & $6.0 \pm 4.0$ & $5.0 \pm 4.0$ & $6.0 \pm 2.5$ & 0.849 \\
\hline BMI (Mean \pm SD) & $26.3 \pm 4.4$ & $26.9 \pm 4.3$ & $24.6 \pm 4.3$ & 0.073 \\
\hline Myocardial infarction & $15(11.1 \%)$ & $12(10.3)$ & $3(15.8)$ & 0.444 \\
\hline Stroke & $14(10.4)$ & $12(10.3)$ & $2(10.5)$ & 0.999 \\
\hline Systemic hypertension & $85(63.0)$ & $74(63.3)$ & $11(57.9)$ & 0.618 \\
\hline Hypercholesterolemia & $58(43.0)$ & $51(44)$ & $7(36.8)$ & 0.561 \\
\hline Hypertriglyceridemia & $35(25.9)$ & $19(25)$ & $6(31.6)$ & 0.544 \\
\hline Smoking & $65(48.1)$ & $56(48.3)$ & $9(47.4)$ & 0.942 \\
\hline Osteoporosis & $20(14.8)$ & $17(14.7)$ & $3(15.8)$ & 0.999 \\
\hline Previous diabetes treatment & $127(94.1)$ & $111(95.7)$ & $16(84.3)$ & 0.084 \\
\hline Age at onset (Mean \pm SD) & $62.4 \pm 12.3$ & $62.6 \pm 12.7$ & $61.5 \pm 11.3$ & 0.742 \\
\hline Years of T2D duration (Mean \pm SD) & $14.7 \pm 11.3$ & $14.6 \pm 11.2$ & $15.5 \pm 12.3$ & 0.714 \\
\hline Insulin use & $23(19.3)$ & $19(18.6)$ & $4(23.5)$ & 0.494 \\
\hline Diabetic nephropathy & $38(28.1)$ & $28(24.1)$ & $10(52.6)$ & 0.010 \\
\hline Diabetic retinopathy & $60(44.4)$ & $50(43.1)$ & $10(52.6)$ & 0.438 \\
\hline Any microvascular complication & $77(57.0)$ & $62(53.4)$ & $15(78.9)$ & 0.037 \\
\hline Vascular problems & $58(43.0)$ & $48(41.4)$ & $10(52.6)$ & 0.358 \\
\hline Urinary incontinence & $24(18.0)$ & $18(15.7)$ & $6(33.3)$ & 0.070 \\
\hline Visual deficit & $34(54.0)$ & $31(57.4)$ & $3(33.3)$ & 0.280 \\
\hline Falls & $63(46.7)$ & $50(43.1)$ & $13(68.4)$ & 0.040 \\
\hline Depression symptoms & $27(20.0)$ & $19(16.4)$ & $8(42.1)$ & 0.009 \\
\hline Frailty & $16(14.0)$ & $11(10.6)$ & $5(50.0)$ & 0.001 \\
\hline Polypharmacy & $78(57.8)$ & $67(57.8)$ & $11(57.9)$ & 0.991 \\
\hline
\end{tabular}

BMI: body mass index; IQR: interquartile range; SD: standard deviation; T2D: type 2 diabetes.

Table 2 presents the univariate logistic regression analyses of CIM. The models found that diabetic nephropathy $(p=0.014)$, having any microvascular complication ( $p=0.046)$, previous falls ( $p=0.047)$, depression symptoms ( $p=0.013$ ), and frailty ( $p=0.011$, pre-frailty was non-significant) were associated with CIM, whereas urinary incontinence did not reach statistical significance ( $p=0.070)$. Conversely, variables such as previous history of myocardial infarction or stroke, hypercholesterolemia, hypertension, hypertriglyceridemia, or smoking were not associated with CIM.

However, the multivariate logistic regression model showed that only urinary incontinence (OR: 13.9; 95\% Cl: 2.12-80.84; $\mathrm{p}<0.001$ ) and frailty (OR: 4.2; 95\% Cl: 0.75-22.74; $\mathrm{p}=0.019$ ) were independently associated with CIM among DM participants (Table 3 ). The model explained $31 \%$ of the variability observed in the composite measure of CIM $\left(R^{2}=0.319\right)$.
Table 2. Univariate regression analysis of variables associated to cognitive impairment in type 2 diabetes participants

\begin{tabular}{|c|c|c|c|}
\hline Variable & OR & $95 \% \mathrm{Cl}$ & $p$ value \\
\hline $\mathrm{BMI}$ & 0.89 & $0.79-1.01$ & 0.077 \\
\hline $\begin{array}{l}\text { Previous myocardial } \\
\text { infarction }\end{array}$ & 0.61 & $0.16-2.42$ & 0.487 \\
\hline Previous stroke & 0.98 & $0.20-4.77$ & 0.981 \\
\hline $\begin{array}{l}\text { Systemic } \\
\text { hypertension }\end{array}$ & 1.28 & $0.48-4.44$ & 0.622 \\
\hline Hypercholesterolemia & 1.34 & $0.49-3.66$ & 0.562 \\
\hline Diabetic nephropathy & 3.49 & $1.29-9.54$ & 0.014 \\
\hline $\begin{array}{l}\text { Any microvascular } \\
\text { complication }\end{array}$ & 3.27 & $1.02-10.44$ & 0.046 \\
\hline Falls & 2.86 & $1.02-8.05$ & 0.047 \\
\hline $\begin{array}{l}\text { Depression } \\
\text { symptoms }\end{array}$ & 3.71 & $1.32-10.45$ & 0.013 \\
\hline Frailty & 4.89 & $1.12-21.30$ & 0.011 \\
\hline Polypharmacy & 1.01 & $0.38-2.69$ & 0.991 \\
\hline Urinary incontinence & 2.69 & $0.90-8.11$ & 0.078 \\
\hline
\end{tabular}

BMI: body mass index; OR: odds ratio. 
Table 3. Multivariate regression analysis of variables associated to cognitive impairment in type 2 diabetes participants

\begin{tabular}{llcc}
\hline Model & \multicolumn{1}{c}{ Variable } & OR $(95 \% \mathrm{Cl})$ & p value \\
\hline 1 & BMI & $0.93(0.74-1.17)$ & 0.538 \\
R2 $=0.363$ & Diabetic nephropathy & $1.57(0.25-9.70)$ & 0.627 \\
& Urinary incontinence & $10.22(1.52-68.82)$ & 0.017 \\
& Frailty & $3.27(0.38-28.38)$ & 0.047 \\
& Depression & $1.21(0.11-13.67)$ & 0.879 \\
\hline 2 & Falls & $1.86(0.30-11.39)$ & 0.503 \\
\hline R2 $=0.313$ & Frailty & $4.12(0.75-22.74)$ & 0.019 \\
\hline
\end{tabular}

BMI: body mass index; OR: odds ratio.

\section{DISCUSSION}

In this sample of community dwelling diabetic elderly persons, urinary incontinence and frailty were independently associated with cognitive impairment. Given that the adequate management of elderly DM patients is dependent on their functional status, investigating the presence of cognitive decline and additional geriatric syndromes within this population is of the utmost importance.

A previous study in elderly Mexican Americans with DM reported that the prevalence of CIM, urinary incontinence, and individual components of the frailty syndrome (muscle strength, slowness) was lower, which contrasts with the data herein reported ${ }^{16}$. However, its definition of cognitive impairment could potentially overestimate the prevalence of CIM (MMSE scores), especially considering that the scores were not adjusted for confounders such as age and schooling in comparison to the present study.

The association of DM and CIM has been consistently shown in several studies; the reported associations suggest a potential role of vascular and non-vascular mechanisms for CIM in DM patients ${ }^{17,18}$. Although the mechanisms of cognitive dysfunction associated to DM are still unclear, combined neurological dysfunction, inflammation, hyperglycemia, insulin resistance, and vascular dysfunction have been proposed to be either causal or contributing elements to the development of both Alzheimer's disease and vascular dementia. Additionally, DM-associated microvascular complications, especially diabetic nephropathy and retinopathy, have also been linked to brain stroke and small-vessel disease associated with cognitive dysfunction ${ }^{19,20}$.
The association of frailty with CIM has been described in other studies ${ }^{21,22}$; however, the association of frailty and CIM in elderly DM patients has not previously been reported. Avila-Funes, et al. proposed that frailty is a major risk factor for the development of cognitive impairment and a possible prodromal stage of vascular dementia ${ }^{21}$. This suggests that, despite the lack of association between the traditional cardiovascular risk factors and CIM in our study, the presence of frailty may account as a mechanism for vascular-mediated cognitive decline. It is important to consider that in other studies, DM has also been associated to an increase in the risk of frailty ${ }^{23}$; moreover, frail DM patients have an increased risk of complications when compared to patients with comorbidities ${ }^{24-27}$. These may play a role as potential confounders to the established association in our study.

Additionally, we show that urinar incontinence was also independently associated with CIM in DM patients; however, a causal link cannot be established to be precise on its association, given that DM is also a known risk factor for the development of urinary incontinence and the latter may already be present before CIM onset. A recent study suggests that biochemical measures, mainly hyperglycemia, are not independent predictors of the presence of urinary incontinence in DM patients ${ }^{28}$. Hsu, et al. investigated the association of risk factors related to the presence of urinary incontinence in elderly DM subjects ${ }^{29}$. They concluded that dependence on ambulation and transferring as well as the presence of CIM is associated with the presence of urinary incontinence in patients with DM, which is consistent with our findings. 
Among the correlates that were associated, though not independently with CIM, microvascular complications are the most extensively studied. Despite evidence of microvascular complications leading to CIM, glucose control has not shown significant benefit on cognitive outcomes ${ }^{19}$. The ACCORD-MIND trial evaluated the cross-sectional and longitudinal association of diabetic retinopathy on cognitive function and brain volume, suggesting that retinal microvascular damage leads to decreased gray matter and cognitive function in diabetic patients, but is not necessarily predictive of vascular-mediated cognitive dysfunction ${ }^{30}$. Our results imply that microvascular complications, in particular diabetic nephropathy, are more frequent in patients with CIM, but their association is not independent. Additionally, microvascular complications are related to age of DM onset and years of DM exposure, both of which were not different between the group with and without CIM. The role of diabetic nephropathy has not been extensively studied and thus requires further longitudinal confirmation.

In DM patients, vascular complications are an important cause of morbidity, and individuals with cardiovascular risk factors are traditionally at higher risk. A large recent study ${ }^{31}$ suggested that metabolic syndrome, central obesity, diabetes mellitus, and other cardiovascular risk factors were associated to incident mild cognitive impairment and dementia; however, this observation is true for patients under the age of 75 years as those included in the population of the study. In populations older than 75 years, such as the mean age of our population, the association of cardiovascular risk factors, including metabolic syndrome, dyslipidemia, central obesity, and hypertension with incident cognitive impairment, has been inconsistent ${ }^{31-33}$. This observation is also supported by the observation that carotid stiffness is associated with cognitive impairment in individuals with diabetes, but was not the mechanism of cognitive dysfunction $^{34}$. In our study, we did not find a significant association of cardiovascular risk factors and cognitive impairment, as has been shown for individuals over 75 years. This suggests that CIM in DM patients is not exclusively vascular-mediated and implies that other factors, such as frailty and urinary incontinence, may contribute to the presence of CIM. Nonetheless, these associations must be confirmed in longitudinal follow-up.
Our study had some limitations. First, this study is a cross-sectional analysis, thus limiting our ability to establish causal associations because we lack the possibility to establish a temporal relation between variables. Furthermore, because only a subset of the studied population completed cognitive evaluation, our study population was reduced and the group with confirmed CIM was relatively small. However, no significant differences were found between the initial sampled group and the analyzed group. Additionally, because our model only explains $31 \%$ of the variability observed in CIM, other variables may contribute as correlates to CIM that were not included in the present model for this work. Subjects classified in the cognitive outcome were not assessed according to the standardized method for CIM. Instead, normative data for performance below the $25^{\text {th }}$ percentile in both the MMSE and in IST were used to identify subjects with CIM. We consider that this global assessment is sufficient for the evaluated spectrum, but the results must be interpreted with caution. Nevertheless, the considered correlates have previously been defined or analyzed in other studies involving the Coyoacán cohort, which makes their definition consistent.

In conclusion, in this cohort of community-dwelling elderly, frailty and incontinence, but not cardiovascular risk factors, are associated with a higher frequency of CIM. Geriatric assessment is not usually sought out during a routine examination by an endocrinologist in an elderly patient with DM. Furthermore, given the strong links of DM and vascular complications, cardiovascular risk factors are classically attributed as the cause of CIM in this population, largely overlooking the effect of geriatric comorbidities. This suggests that the contribution of geriatric conditions to the presence CIM in DM patients should be further investigated. Intentional evaluation of these conditions might be of importance to improve patient care and management of the elderly patient with DM and cognitive decline.

\section{ACKNOWLEDGEMENTS}

This study was conducted as part of the Mexican Study of Nutritional and Psychosocial Markers of Frailty among Community-Dwelling Elderly. This project was funded by CONACyT (grant SALUD-2006-C01-45075), Mexico. 


\section{REFERENCES}

1. Aguilar-Salinas CA, Velazquez Monroy O, Gómez-Pérez FJ, et al. Characteristics of patients with type 2 diabetes in México: Results from a large population-based nationwide survey. Diabetes Care. 2003;26:2021-6.

2. Rojas-Martínez R, Aguilar-Salinas CA, Jiménez-Corona A, Gómez-Pérez FJ, Barquera S, Lazcano-Ponce E. Prevalence of obesity and metabolic syndrome components in Mexican adults without type 2 diabetes or hypertension. Salud Publica Mex. 2012;54:7-12

3. Wilson RS, Barnes LL, Krueger KR, Hoganson G, Bienias JL, Bennett DA. Early and late life cognitive activity and cognitive systems in old age. J Int Neuropsychol Soc. 2005;11:400-7.

4. Arvanitakis Z, Wilson RS, Bennett DA. Diabetes mellitus, dementia, and cognitive function in older persons. J Nutr Health Aging. 2006;10:287-91.

5. Mayeda ER, Haan MN, Yaffe K, Kanaya AM, Neuhaus J. Does type 2 diabetes increase rate of cognitive decline in older Mexican Americans? Alzheimer Dis Assoc Disord. 2015;29:206-12.

6. Bangen KJ, Gu Y, Gross AL, et al. Relationship between type 2 diabetes mellitus and cognitive change in a multiethnic elderly cohort. J Am Geriatr Soc. 2015;63:1075-83.

7. Sadanand S, Balachandar R, Bharath S. Memory and executive functions in persons with type 2 diabetes: a meta-analysis. Diabetes Metab Res Rev. 2016;32:132-42.

8. Rosso AL, Eaton $C B$, Wallace $R$, et al. Combined impact of geriatric syndromes and cardiometabolic diseases on measures of functional impairment. J Gerontol A Biol Sci Med Sci. 2011;66:349-54.

9. Lu FP, Lin KP, Kuo HK. Diabetes and the risk of multi-system aging phenotypes: a systematic review and meta-analysis. PLoS One. 2009;4:e4144

10. Mokri H, Avila-Funes JA, Meillon C, Gutiérrez Robledo LM, Amieva H. Normative data for the Mini-Mental State Examination, the Free and Cued Selective Reminding Test and the Isaacs Set Test for an older adult Mexican population: the Coyoacán Cohort Study. Clin Neuropsychol. 2013;27:1004-18.

11. Fried LP, Tangen CM, Walston J, Newman AB, Hirsch C, Gottdiener J. Frailty in older adults: evidence for a phenotype. J Gerontol A Biol Sci Med Sci. 2001;56:M146-56.

12. Avila-Funes JA, Medina-Campos RH, Tamez-Rivera O. Frailty is associated with disability and recent hospitalization in community-dwelling elderly: The Coyoacan Cohort. J Frailty Aging. 2014;3:206-10.

13. Inouye S, Studenski S, Tinetti M, Kuchel GA. Geriatric syndromes: Clinical, research and policy implications of a core geriatric concept. J Am Geriatr Soc. 2007;55:780-91.

14. Sheikh JL, Yesavage JA. Geriatric Depression Scale (GDS). Recent evidence and development of a shorter version. Clin Gerontol. 1986;5:165-72.

15. Walckiers D, Van der Heyden J, Tafforeau J. Factors associated with excessive polypharmacy in older people. Arch Public Health. 2015;73:50.

16. Beard HA, Markides KS, Al Ghatrif M, Kuo YF, Raji MA. Trends in diabetes medication use and prevalence of geriatric syndromes in older Mexican Americans from 1993/1994 to 2004/2005. Ann Pharmacother. 2010;44:1376-83.
17. Luchsinger JA. Type 2 diabetes, related conditions, in relation and dementia: an opportunity for prevention? J Alzheimers Dis. 2010;20:723-36

18. Abbatecola AM, Paolisso G, Sinclair AJ. Treating diabetes mellitus in older and oldest old patients. Curr Pharm Des. 2015;21:1665-71.

19. Umegaki $H$. Type 2 diabetes as a risk factor for cognitive impairment: current insights. Clin Interv Aging. 2014;9:1011-19.

20. de Bresser J, Reijmer YD, van den Berg E. Microvascular determinants of cognitive decline and brain volume change in elderly patients with type 2 diabetes. Dement Geriatr Cogn Disord. 2010;30:381-6.

21. Avila-Funes JA, Carcaillon L, Helmer C, et al. Is frailty a prodromal stage of vascular dementia? Results from the Three-City Study. J Am Geriatr Soc. 2012;60:1708-12.

22. Avila-Funes JA, Amieva $H$, Barberger-Gateau $P$, et al. Cognitive impairment improves the predictive validity of the phenotype of frailty for adverse health outcomes: the three-city study. J Am Geriatr Soc. 2009;57:453-61.

23. García-Esquinas E, Graciani A, Guallar-Castillón P, López-García E, Rodríguez-Mañas L, Rodríguez-Artalejo F. Diabetes and risk of frailty and its potential mechanisms: a prospective cohort study of older adults. J Am Med Dir Assoc. 2015;16:748-54.

24. Hubbard RE, Andrew MK, Fallah N, Rockwood K. Comparison of the prognostic importance of diagnosed diabetes, co-morbidity and frailty in older people. Diabet Med. 2010;27:603-6.

25. Cobo A, Vázquez LA, Reviriego J, Rodríguez-Mañas L. Impact of frailty in older patients with diabetes mellitus: An overview. Endocrinol Nutr. 2016;63:291-303

26. Cacciatore F, Testa G, Galizia G. Clinical frailty and long-term mortality in elderly subjects with diabetes. Acta Diabetol. 2013;50:251-60.

27. Umegaki H. Sarcopenia and frailty in older patients with diabetes mellitus. Geriatr Gerontol Int. 2016;16:293-9.

28. Weinberg AE, Leppert JT, Elliott CS. Biochemical measures of diabetes are not independent predictors of urinary incontinence in women. J Urol. 2015;194:1668-74.

29. Hsu A, Conell-Price J, Stijacic Cenzer I, et al. Predictors of urinary incontinence in community-dwelling frail older adults with diabetes mellitus in a cross-sectional study. BMC Geriatr. 2014:14:137.

30. Hugenschmidt CE, Lovato JF, Ambrosius WT, Bryan RN, Gerstein $\mathrm{HC}$, Horowitz KR, et al. The cross-sectional and longitudinal associations of diabetic retinopathy with cognitive function and brain MRI findings: the Action to Control Cardiovascular Risk in Diabetes (ACCORD) trial. Diabetes Care. 2014;37:3244-52

31. Ng TP, Feng L, Nyunt MS, Feng L, Gao Q, Lim ML. Metabolic syndrome and the risk of mild cognitive impairment and progression to dementia: Follow-up of the Singapore Longitudinal Ageing Study Cohort. JAMA Neurol. 2016;73:456-63.

32. Solfrizzi $V$, Scafato $E$, Capurso $C$, et al. Metabolic syndrome, mild cognitive impairment, and progression to dementia. The Italian Longitudinal Study on Aging. Neurobiol Aging. 2011;32:1932-41.

33. van den Berg E, Biessels GJ, de Craen AJ, Gussekloo J, Westendorp RG. The metabolic syndrome is associated with decelerated cognitive decline in the oldest old. Neurology. 2007;69:979-85.

34. Geijselaers SL, Sep SJ, Schram MT, van Boxtel MP, van Sloten TT, Henry RM. Carotid stiffness is associated with impairment of cognitive performance in individuals with and without type 2 diabetes. The Maastricht Study. Atherosclerosis. 2016;253:186-93. 